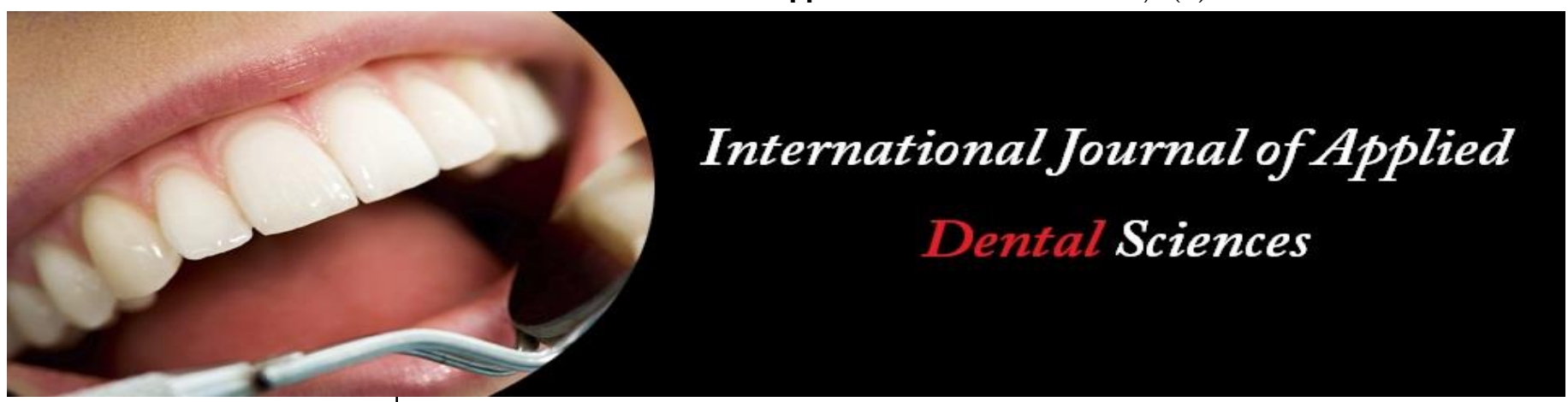

ISSN Print: 2394-7489

ISSN Online: 2394-7497

IJADS 2020; 6(3): 474-479

(C) 2020 IJADS

www.oraljournal.com

Received: 26-03-2020

Accepted: 15-05-2020

Dr. Lobna Mohammed Ziad Al Jabi

PhD. Student. Department of Removable Prosthodontics. Faculty of Dentistry. Hama University, Syria

Dr. Hussein Ali Al Essa PhD Holder. Assistant Professor. Department of Removable Prosthodontics. Faculty of Dentistry. Hama University, Syria

Dr. Samer Kamel Ibrahim PhD Holder. Professor. Department of Microbiology. Faculty of Veterinary Medicine. Hama University, Syria

Dr. Raeef abdulghani Al Najjar PhD. Student. Department of Removable Prosthodontics. Faculty of Dentistry. Hama University, Syria
Corresponding Author: Dr. Lobna Mohammed Ziad Al Jabi

PhD. Student. Department of Removable Prosthodontics. Faculty of Dentistry. Hama University, Syria

\section{Evaluating the growth of candida albicans on removable complete dentures bases manufactured using CAD/CAM designed metal (Co-Cr casted from milled wax patterns) and injection molded resin}

\author{
Dr. Lobna Mohammed Ziad Al Jabi, Dr. Hussein Ali Al Essa, Dr. Samer \\ Kamel Ibrahim and Dr. Raeef abdulghani Al Najjar
}

DOI: https://doi.org/10.22271/oral.2020.v6.i3g.995

\begin{abstract}
Acrylic resin processed by compression molded technique had been used to manufacture the removable complete dentures bases for many years.

However there are many disadvantages connected to the use of this material with the ordinary compression molding as a denture base, the reason that made the researches try to find a better alternatives whether by changing the processing technique: like in the case of using acrylic resins processed by injection molded technique which proved to be a better choice in many aspects, on the other hand some researchers preferred to use another material so they used metal bases as replacement for the ordinary acrylic denture base. There are many ways to make patterns for metal bases casting in partial and complete dentures.

Recently many researchers and practitioners used CAD-CAM technologies to make patterns for metal casting and facilitate the work, and save time.

This study compares the candida albicans colony formation on the two materials mentioned above as an advantage to the material studied.

Purpose: The purpose of this study is to compare the growth of candida albicans on removable complete dentures bases manufactured using CAD/CAM designed metal ( $\mathrm{Co}-\mathrm{Cr}$ casted from milled wax patterns) and injection molded acrylic resin.

Materials and Methods: Split-palate maxillary complete dentures were fabricated for 10 edentulous patients. one-half of the palate was made in acrylic resin and the other half in $\mathrm{Co}-\mathrm{Cr}$ metal palate that was casted from CAD-CAM milled patterns for each patient in this study.

specimens were collected at 1,3 , and 5 months using a modified imprint culture method. Speciation of Candida albicans was performed using a chromogenic culture medium. Two-sample t-test was employed to determine effects and significant interactions between the acrylic and the metal groups.

Results: Significant differences were observed in the candida albicans colony counts between both materials during the first, the third, and the fifth month. The Colony forming unit $(\mathrm{CFU}) / \mathrm{cm}^{2}$ means were significantly less on the Co-Cr casted metal denture bases than $\mathrm{CFU} / \mathrm{cm}^{2}$ means on the injection molded acrylic resin.

Conclusions: The metal base proved to be effective in decreasing the candida albicans growth typically present in complete dentures. Colony counts of Candida albicans were higher in the injection molded acrylic resin part of denture bases of the patients in this study.
\end{abstract}

Keywords: Injection molded resin, CAD, CAM, Metal, Cobalt, Chrome, Wax Milling, Denture base, candida albicans, chrome agar, imprint culture, $\mathrm{CFU}$

\section{Introduction}

Dr. Walter Wright (1937) introduced Polymethyl methacrylate as a denture base material which became the major polymer to be used. Since ages, polymethyl methacrylate (PMMA) has been used to fabricate the dentures, and since its introduction as a denture base material, its use has become almost universal ${ }^{[1-5]}$.

On the other hand, this material have many disadvantages as a denture base, such as dimensional changes ${ }^{[2,3,6,7]}$, residual monomer allergy ${ }^{[5]}$, low thermal conductivity ${ }^{[5,8]}$, low hardness ${ }^{[5,8]}$, water sorption ${ }^{[9]}$, The lack of dimensional stability ${ }^{[2]}$. 
Dentures are usually subjected to a combination of compressive, tensile, shearing loads and these forces are increased in ill-fitting dentures and these forces are traumatic to both soft and hard tissue of denture bearing surface ${ }^{[10]}$.

\section{Injection molded resin as a denture base replacement}

Attempts to overcome the problems associated with the use of compression molding technique have resulted in the development of the continuous injection system. Introduced in 1942, continuous injection molding eliminates the flash of resin between the halves of the flask and compensates for polymerization shrinkage by forcing extra resin into the flask during polymerization.

The results of Nogueira S.S et.al in the year 1999 study indicated a significantly higher degree of accuracy for the injection molding technique for processing dentures over the standard compression molding technique. They also concluded that the injection molding system would save time in construction of dentures because of the smaller incisal pin opening and the reduction in time consumed in postprocessing adjustments ${ }^{[11]}$.

Dentures that have been processed by the compression molded technique show greater changes in a horizontal and diagonal direction than dentures processed by the injection molded technique ${ }^{[12]}$. Injection molding allows directional control of the polymerization process through the flask design. A constant flow of new material from the sprue compensates for the polymerization shrinkage. Various injection-molded denture base materials and processing techniques are now available, each claiming to produce more accurate denture bases. The SR-Ivocap system (Ivoclar AG, Schaan, Liechtenstein) is an injectable PMMA. There have been various studies carried out on the material, and the literature in general supports the claim that the SR-lvocap system has less linear dimensional change than conventional PMMA ${ }^{[13]}$. Injection molded resin is superior in terms of dimensional accuracy compared to conventional pressure packed PMMA resin ${ }^{[14]}$.

A smooth denture surface will be beneficial to the patient and contribute to a healthier oral environment. Microorganisms are a normal part of the oral cavity; however, any effort by the dentist to reduce the likelihood of plaque and biofilm accumulation will benefit the patient immensely ${ }^{[15]}$.

Berger and his colleagues found in their research in 2006 that injection molded resin showed the least rough specimen among all those tested in that study (heat cured injection molded, heat cured compression molded, Autopolymerized, Microwave polymerized) ${ }^{[15]}$.

Surface roughness may contribute to the rate of microbial colonization and biofilm formation on acrylic resin.

Studies by Morgan and Wilson and by Lamfon showed that bacteria and fungus have more of a propensity to adhere to rough acrylic resins ${ }^{[15-17]}$.

\section{Metal alloys as a denture base replacement}

PMMA denture bases have good mechanical, biological and esthetic properties but they may fail because of excessive masticatory or functional forces. In such circumstances metal denture base can be used ${ }^{[18,6,19]}$.

Various studies have shown that metal dentures were perceived as more comfortable than acrylic resin denture reduces burning sensation, allergic reactions, eliminating microbial colonization, is fracture resistant, thin, comfortable to the patient and gives them a feeling of chewing food naturally ${ }^{[20]}$. Acrylic denture base materials may serve as a reservoir for microorganisms like candida albicans and bacteria ${ }^{[21,22]}$.

In spite of many advantages, metal denture bases do not enjoy widespread use in clinical practice. Metal based dentures are more retentive, have less Occlusal discrepancy, cause fewer sore spots, have a reduced incidence of fracture, feel better to the patient, are better thermal conductors, act as a stable record base, have a thinner palate that aids speech, better preserve the residual alveolar ridge, are less porous, deform less during lateral mandibular function, and are more accurate in tissue detail ${ }^{[18]}$

Metal denture base are more tissue tolerant and resistant to deformation than acrylic denture base ${ }^{[6]}$.

The coefficient of thermal conductivity of PMMA is approximately $0.2 \mathrm{~W} / \mathrm{min}^{\circ} \mathrm{K}$; this rate is almost one-third of the coefficient of thermal conductivity of most metals. Due to this difference, in some studies, acrylic denture base has been replaced with metal base ${ }^{[8]}$.

For metallic prostheses, the traditional lost-wax casting technique is most commonly used in dentistry. Defects and inaccuracy generated in this labor-intensive casting process, that can take 1 week to complete, call for new methods to satisfy customer needs nowadays.

Recent research achievements in the areas of computer-aided design and computer aided manufacturing (CAD/CAM) technology have created alternative routes to fabricate dental prostheses and dental implants ${ }^{[23]}$.

Digital strategies widen the scope of therapeutic applications for partial dentures as a result of improved design and production control, new materials, and improved efficiencies that will likely enhance outcomes and improve patient experiences ${ }^{[24,25]}$.

\section{Candida albicans growth on dentures bases}

Dentures create an environment that helps the localization and development of potentially virulent organisms.

Factors such as prosthesis fit, hygiene, and host susceptibility contribute to the development and progression of this condition so that the reported prevalence ranges between $10 \%$ and $67 \%$ in complete denture wearers among several populations and age groups ${ }^{[26]}$. The impression surface of a maxillary denture in particular may be a common reservoir for microorganisms. Candida albicans is the most important and predominant oral fungal pathogen. It has the ability to adhere and proliferate on both soft and hard tissues, forming complex biofilm structures. This depends on initial attachment to the denture impression surface, which in turn depends on physical properties of the material surface such as porosity, surface free energy, hydrophobicity, and roughness. These variables are all influenced by type of the materials used in preparing dentures, their polymerization method, and any surface modifications in corporation of fibers or surface coating ${ }^{[28,27]}$.

Most manifestations of candidiasis are associated with the formation of Candida albicans biofilms on surfaces such as prostheses. Biofilms in denture plaque represent a protective reservoir for oral microbes.

There is a strong possibility that the colonization of denture bearing surfaces can serve as a reservoir for disseminated infections. In addition surface irregularities could increase the likelihood of residual microorganisms on the surface after the prosthesis has been cleaned, thus allowing continuous reinfection of the palate ${ }^{[29]}$.

Microorganisms are a normal part of the oral cavity; however, any effort by the dentist to reduce the likelihood of plaque and 
biofilm accumulation will benefit the patient immensely ${ }^{[15]}$. Injection molded resin material show less plaque adhesion; therefore, less denture induced stomatitis is probable in patients wearing dentures made with this technique ${ }^{[27]}$.

Metal denture base is effective in decreasing fungal growth in complete dentures and provides to be an alternative dental service for edentulous patients ${ }^{[29]}$.

\section{Materials and Methods}

Ten maxillary edentulous patients (with no systemic health problems, e.g.: diabetes or smoking habits) were selected from the prosthodontics department Clinic (Faculty of dentistry-Hama university Syria). All patients agreed with the trial protocol and signed a voluntary consent agreement. Maxillary complete dentures were constructed using a splitpalate technique (Fig 1), the patients final casts were scanned with laboratory scanner (Edge scanner, DOF, Korea), and the scanned Stl file then transferred to the design software (3SHAPE Complete Restorative Software, Denmark) to be used in designing the complete maxillary plate patterns, then after completing the designing process, the design Stl file was send to DG shape software to be milled from a wax blank (Diamond, Canada) using the Roland DWX-52D 5-Axis Dental Milling Machine milling machine( Roland, USA).

All dentures were made using Cobalt-Chrome partial denture alloy a (Wironit extra-hard-Bego- Germany) on the right half of the denture palate according to the manufacturer instructions.

The left half of the denture palate were made of injection molded acrylic resin (Ivoclar vivadent- Liechtenstein).

A protocol of oral hygiene and home care of the prostheses was implemented to minimize the variables normally seen in clinical studies.

Sampling collection was performed at 1, 3, and 5 months post-insertion of dentures.

Sampling collection was performed using a modified imprint culture method: $2.0 \mathrm{~cm} 2$ sterile sponges pre-moistened with $10 \mathrm{ml}$ Buffered peptone water (Liofilchem, Italy) were placed for 60 seconds on each intaglio surface. Each sponge was then placed into individual tubes containing $10 \mathrm{ml}$ of Buffered peptone water. The tubes were mechanically stirred for 15 seconds in Multi Shaker (Tokyo Rikakikai Co Ltd, Japan). Using a pipette, $0.1 \mathrm{ml}$ solution was transferred to the chromogenic agar (CHROMagar Candida, Liofilchem, Italy). The solution was then spread evenly over the surface of the plates and transferred for incubation at $37^{\circ} \mathrm{C}$ for 48 hours. Candida albicans colonies on each media were identified because of its green color according to the manufacturers' instructions and manuals counted and expressed as total colony forming units per $\mathrm{cm}^{2}\left(\mathrm{CFU}\right.$ per $\left.\mathrm{cm}^{2}\right)$. Colony counting was done manually and to confirm the results the count was done again using Open CFU software.

The differentiation of Candida albicans species such as albicans, and other types of candida albicans was possible because these species grew as different colors of isolated colonies on the agar surface, and the candida albicans colonies were green in color according to CHROMagar manufacturer manual.

This study took place at the Department of Prosthodontics, Faculty of Dentistry and in the lab of scientific Research, Faculty of Veterinary medicine, Hama University- Syria.

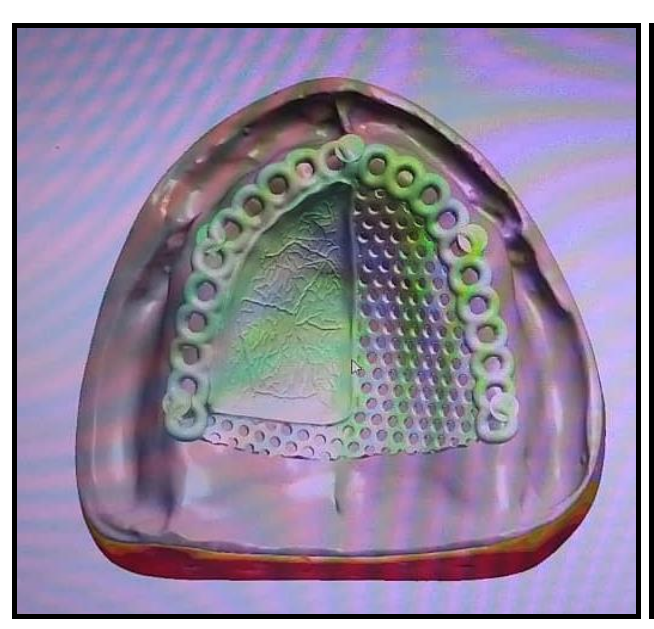

Fig 1: Digital design

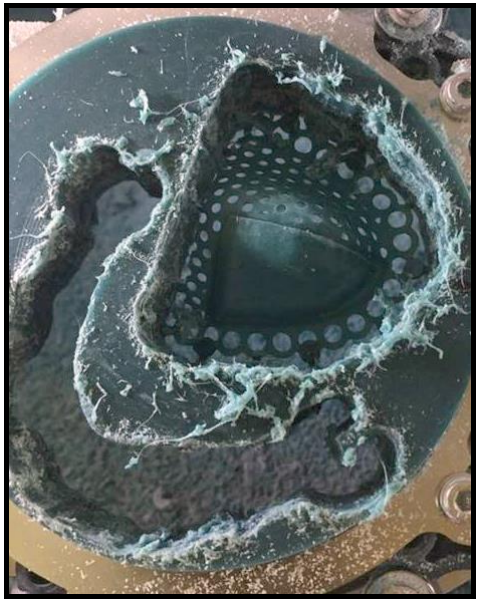

Fig 2: Milled wax blank

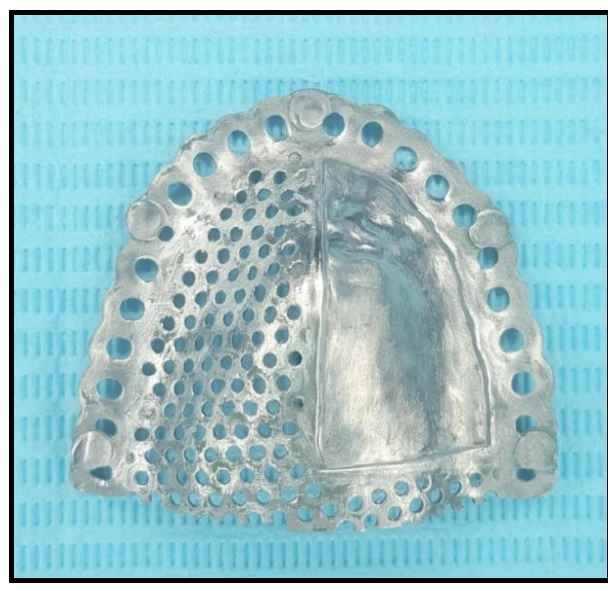

Fig 3: Metal palate after casting

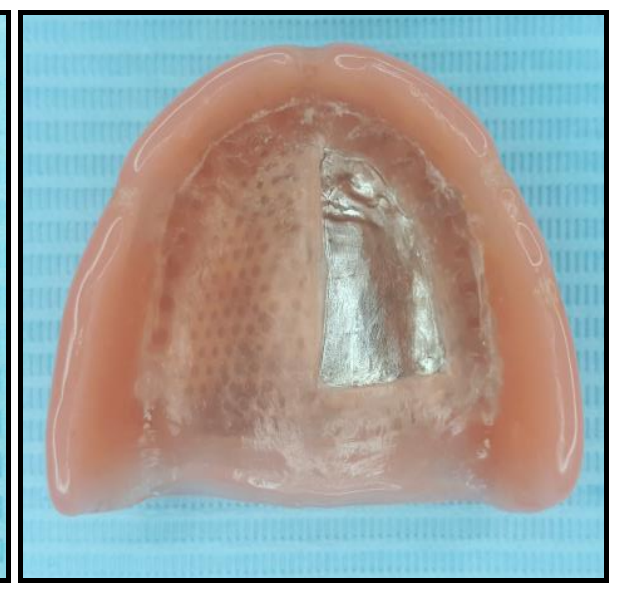

Fig 4: Maxillary denture intaglio surface 


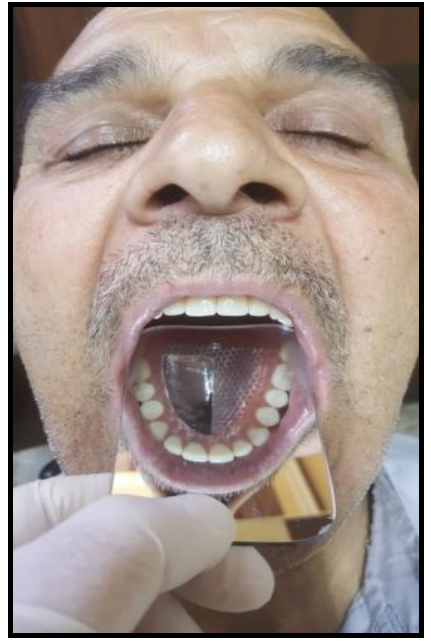

Fig 5: Maxillary denture in the oral cavity

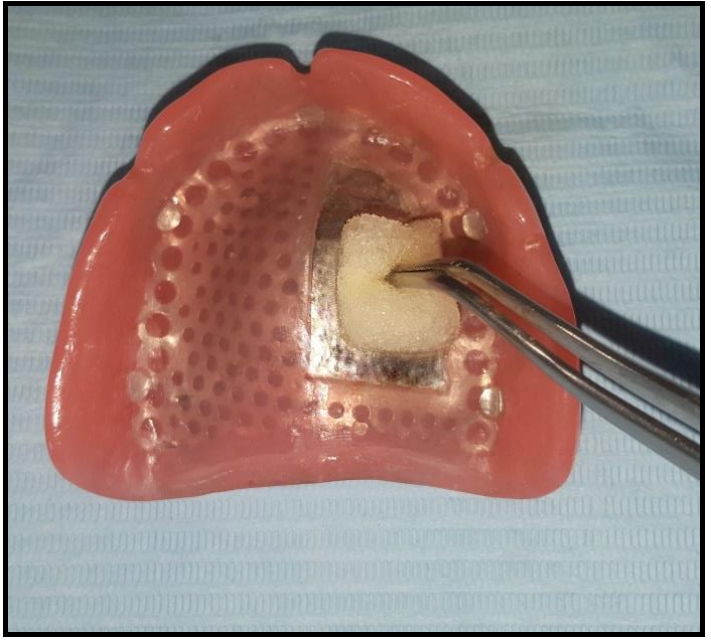

Fig 6: Imprint sampling from the metal half

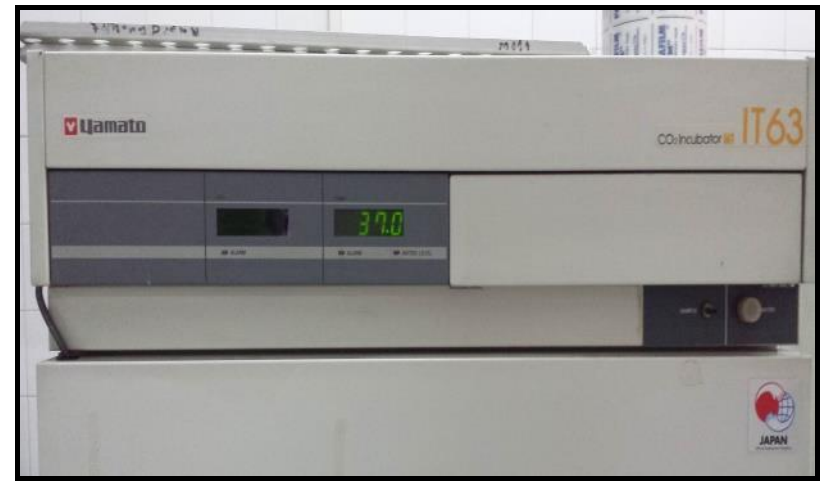

Fig 5: Incubating plates in $37^{\circ} \mathrm{C}$ Incubator

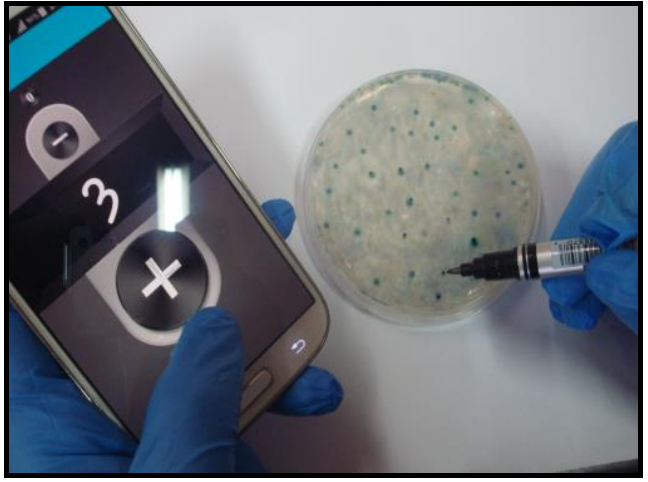

Fig 6: Manual CFU counting

\section{Statistical study}

A two-sample t-test for independent variables was employed to determine the effects and significant interactions between the $\mathrm{CFU} / \mathrm{Cm}^{2}$ in the injection molded acrylic resin and the $\mathrm{Co}-\mathrm{Cr}$ casted metal half. The significant differences in the average values, and all values were considered significant at $\mathrm{P}$ $\leq 0.05$.

\section{Results}

Table 1: candida growth comparison between metal bases and injection molded resin expressed by $\mathrm{CFU} / \mathrm{cm}^{2}$

\begin{tabular}{|c|c|c|c|c|c|c|}
\hline \multicolumn{5}{|c|}{ Candida Albicans Candida Colonies CFU/cm2 } \\
\hline Test Time & One month & Three months & Five months & One month & Three months & Five months \\
\hline Mean & 170 & 300 & 370 & 295 & 395 & 560 \\
\hline SD & 45.82 & 50 & 67.82 & 47.16 & 41.53 & 43.58 \\
\hline
\end{tabular}

There were significant differences in candida albicans colony count (the $\mathrm{CFU} / \mathrm{cm}^{2)}$ between the metal half and the injection

molded resin half of the dentures.

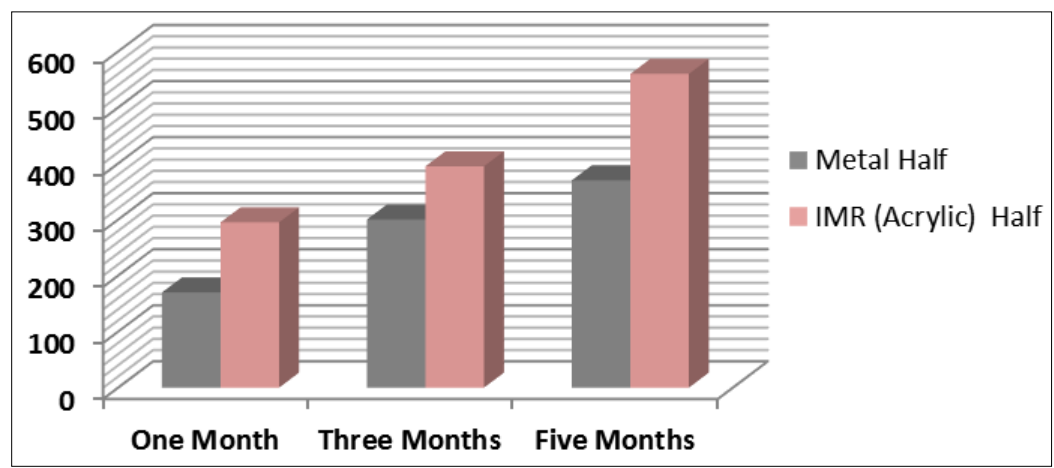

Graph 1: candida growth comparison between metal bases and injection molded resin expressed by CFU/cm ${ }^{2}$ 


\section{Discussion}

Candida albicans was studied in this article because it's the main isolate from denture stomatitis lesions ${ }^{[30]}$.

The samples were taken from the inner surface of the dentures because $\mathrm{C}$. albicans is found in higher numbers on the fitting surface of the prosthesis than on the infected mucosa, hence the role of the prosthesis as a reservoir of infection ${ }^{[31]}$.

Injection molded resin had been used in this study, because of its more accurate than compression molded denture acrylics [11], and injection molded resin shows less dimensional changes than the compression molded [12-14] and most importantly the injection molded resin have smoother surface than the compression molded one ${ }^{[15]}$ which can affect directly the attachment of microorganisms including candida to the surfaces of the dentures, because smoother the surface less microorganisms can colonize it ${ }^{[15-17]}$.

The CAD/CAM technique was used to mill the wax patterns because it's easier to design the palate pattern using this technique, and it's more likely to get similar surface features by using the same wax blank and the milling machine, with the same casting steps for all the patients.

This technique also can save time and labor ${ }^{[32]}$.

Co-Cr casted metal palate had been used in this study also because it considered to be a good replacement for the ordinary compression molded acrylic resin because it is fracture resistant and its more comfortable than acrylic resin denture, reduces burning sensation, allergic reactions, and most importantly it eliminating microbial colonization ${ }^{[20]}$.

Due to the surface irregularities of acrylic resin, a factor in the entrapment of microorganisms, there is a need for improved denture base materials that could inhibit the growth of microorganisms ${ }^{[29]}$.

As for this study results, it was found that $\mathrm{Co}-\mathrm{Cr}$ casted metal palate is less in candida albicans colonization than the injection molded resin over time, which agrees with Pryor who noted an inhibitory action on the growth of microorganisms when using metal bases ${ }^{[33]}$, it also agrees with the results of Perezous. L and her colleagues in their research 2006, but in their research they compare candidal growth on compression molded acrylic resin and metal palates made of nickel-chromium-beryllium alloy and the patients were aids patients ${ }^{[29]}$.

The metal base complete dentures in this study proved to be effective in decreasing the candida albicans growth typically associated with these prostheses.

This happens maybe because metal surfaces are less rough than the acrylic resin surfaces ${ }^{[34]}$, which can affect directly the attachment of microorganisms including candida to the surfaces of the dentures ${ }^{[15-17]}$.

Also it maybe occurred because metal denture bases have better dimensional accuracy than the acrylic resin ones ${ }^{[35,36]}$ poor fitting dentures may can be connected to the candidal accumulation ${ }^{[37]}$.

Also some studies shows that $\mathrm{Co}-\mathrm{Cr}$ metal denture bases can form less passive film. The relative permittivity level of the passive film on the $\mathrm{Co}-\mathrm{Cr}$ alloy is considerably lower compared to other alloys which makes the level of adherent biofilm on the Co-Cr lower ${ }^{[38]}$.

Also it may relate to the lower surface free energy of the Co$\mathrm{Cr}$ denture base alloys when compared with heat cure acrylic compression molded resin and cold cure injection molded resin ${ }^{[39]}$. Which can lower plaque accumulation and lower the adhesion of candida albicans ${ }^{[40,41]}$.

\section{Conclusions}

- Within the limits of this study, it was concluded that the candida albicans growth at all-time intervals studied was less on the Co-Cr maxillary metal denture bases casted from milled wax patterns, than that was on the injection molded acrylic resin.

- Metal base prostheses may be of clinical significance as a treatment alternative to patients who are prone to higher incidence of candidal infections.

\section{Ethical Approval}

All participants signed consent forms prior to the study. The right of the participants to withdraw at any time was explained and preserved during the study.

\section{Acknowledgement}

The authors would like the following labs for their outstanding help and cooperation:

AL BASH Dental Laboratory- D.T. Youssef Mahmoud Bash Aleppo- Syria.

AL SABBAGH Dental Laboratory- D.T Ahmad Mohammed Yasser Al Sabbagh- Hama-Syria.

AL Hindi Dental Laboratory- Eng.: Anas Ahmad BangalyHama- Syria.

\section{References}

1. Sharma A, Shashidhara H. A review: Flexible removable partial dentures. J Dent Med Sci. 2014; 13(12):58-62.

2. Duymus ZY, Yanikoglu ND. Influence of a thickness and processing method on the linear dimensional change and water sorption of denture base resin. Dental Materials Journal. 2004; 23(1):8-13.

3. Park H.-W, Kim C.-W, Kim Y.-S. A comparative study on the accuracies of resin denture bases and metal denture bases. J Korean Acad Prosthodont. 2001; 39(3):250-9.

4. Tandon R, Gupta S, Agarwal SK. Denture base materials: From past to future. Indian J Dent Sci. 2010; 2(2):33-9.

5. Nandal S et al. New era in denture base resins: a review. Dental Journal of Advance Studies. 2013; 1(03):136-143.

6. Rathee M, Bhoria M, Boora P. International Journal of Research in Dentistry Oral Rehabilitation with Metal Base Complete Denture for Patient with Oral Lichen Planus. 2014; 4(4):117-122.

7. Woelfel JB, Paffenbarger GC, Sweeney WT. Dimensional changes occurring in dentures during processing. The Journal of the American Dental Association. 1960; 61(4):413-430.

8. Ghafari T, Hamedi RF, Ezzati B. Does addition of silver nanoparticles to denture base resin increase its thermal conductivity? 2014.

9. Shah $\mathrm{J}$ et al. Comparative evaluation of sorption, solubility and microhardness of heat cure polymethylmethacrylate denture base resin \& flexible denture base resin. Journal of clinical and diagnostic research: JCDR. 2014; 8(8):ZF01.

10. Lechner SK, Thomas GA. Changes caused by processing complete mandibular dentures. The Journal of Prosthetic Dentistry. 1994; 72(6):606-613.

11. Nogueira SS, Ogle RE, Davis EL. Comparison of accuracy between compression-and injection-molded complete dentures. The Journal of prosthetic dentistry. 1999; 82(3):291-300.

12. Garfunkel E. Evaluation of dimensional changes in complete dentures processed by injection-pressing and the pack-and-press technique. Journal of Prosthetic Dentistry. 1983; 50(6):757-761. 
13. Parvizi A et al. Comparison of the dimensional accuracy of injection-molded denture base materials to that of conventional pressure-pack acrylic resin. Journal of Prosthodontics: Implant, Esthetic and Reconstructive Dentistry. 2004; 13(2):83-89.

14. Ahmed M. Comparative adaptation accuracy of heat cured and injection molded resin denture base materials. Journal of Applied Sciences Research. 2012; 8(8):46914696.

15. Berger JC et al. Surface roughness of denture base acrylic resins after processing and after polishing. Journal of Prosthodontics: Implant, Esthetic and Reconstructive Dentistry. 2006; 15(3):180-186.

16. Lamfon $\mathrm{H}$. Investigation into the formation and susceptibility of Candida albicans biofilms and denture plaque in vitro. University of London, 2005.

17. Morgan T, Wilson M. The effects of surface roughness and type of denture acrylic on biofilm formation by Streptococcus oralis in a constant depth film fermentor. Journal of applied microbiology. 2001; 91(1):47-53.

18. Belfiglio EJ. Using metal bases in making complete dentures. Journal of Prosthetic Dentistry. 1987; 58(3):314-317.

19. Ohkube C, Abe M, Hosoi T et al. comparative study of maxillary complete dentures constructed of metal base and metal structure framework. Journal of oral rehabilitation. 2001; 28(1):49-56.

20. Hummel SK et al. A pilot study to evaluate different palate materials for maxillary complete dentures with xerostomic patients. Journal of prosthodontics. 1999; 8(1):10-17.

21. Gomaa FAM, Helal ZH. Isolation and Identification of Microorganisms Associated With Removable Denture: Prevalence of Non Oral Pathogens., Egypt. Acad. J Biolog. Sci, 2010.

22. O'Donnell LE et al. Dentures are a reservoir for respiratory pathogens. Journal of Prosthodontics. 2016; 25(2):99-104.

23. Wu J et al. A feasibility study on laser rapid forming of a complete titanium denture base plate. Lasers in medical science. 2010; 25(3):309-315.

24. Campbell SD et al. Removable partial dentures: The clinical need for innovation. The Journal of prosthetic dentistry. 2017; 118(3):273-280.

25. AlHelal A et al. Comparison of retention between maxillary milled and conventional denture bases: a clinical study. The Journal of prosthetic dentistry. 2017; 117(2):233-238.

26. Pusateri CR, Monaco EA, Edgerton M. Sensitivity of Candida albicans biofilm cells grown on denture acrylic to antifungal proteins and chlorhexidine. Archives of oral biology. 2009; 54(6):588-594.

27. Aslanimehr $\mathrm{M}$ et al. Comparison of Candida Albicans adherence to conventional acrylic denture base materials and injection molding acrylic materials. Journal of Dentistry. 2017; 18(1):61.

28. Yildirim $\mathrm{M}$ et al. Adherence of Candida albicans to glow-discharge modified acrylic denture base polymers. Journal of oral rehabilitation. 2005; 32(7):518-525

29. Perezous LF et al. The Effect of Complete Dentures with a Metal Palate on Candida Species Growth in HIV-Infected Patients. Journal of prosthodontics. 2006; 15(5):306-315.

30. Gacon I, Loster JE, Wieczorek A. Relationship between oral hygiene and fungal growth in patients: users of an acrylic denture without signs of inflammatory process. Clinical Interventions in Aging. 2019; 14:1297.

31. Verran J, Lees G, Shakespeare AP. The effect of surface roughness on the adhesion of Candida albicans to acrylic. Biofouling. 1991; 3(3):183-191.

32. Al Essa HA. CAD/CAM in prosthodontics: A gate to the future. International Journal of Applied Dental Sciences. 2019; 5(3):394-397.

33. Pryor W. Swaged denture bases. The Journal of the American Dental Association. 1922-1928; 15(7):12811288.

34. Nishioka $\mathrm{M}$ et al. Influence of polishing of denture base resin and metal surfaces on wettability with water and saliva. Dental Materials Journal. 2006; 25(1):161-165.

35. Moore F. Organic or metal bases for dentures. The Journal of prosthetic dentistry. 1967; 17(3):227-231.

36. Arafa KA. Effect of different denture base materials and changed mouth temperature on dimensional stability of complete dentures. International Journal of Dentistry. 2016.

37. Coco B et al. Mixed Candida albicans and Candida glabrata populations associated with the pathogenesis of denture stomatitis. Oral microbiology and immunology. 2008; 23(5):377-383.

38. Urushibara Y et al. An analysis of the biofilms adhered to framework alloys using in vitro denture plaque models. Dental Materials Journal. 2014; 33(3):402-414.

39. Sipahi C, Anil N, Bayramli E. The effect of acquired salivary pellicle on the surface free energy and wettability of different denture base materials. Journal of dentistry. 2001; 29(3):197-204.

40. Minagi $\mathrm{S}$ et al. Hydrophobic interaction in Candida albicans and Candida tropicalis adherence to various denture base resin materials. Infection and immunity. 1985; 47(1):11-14.

41. Quirynen M et al., The influence of surface free energy and surface roughness on early plaque formation: an in vivo study in man. Journal of clinical periodontology. 1990; 17(3):138-144. 